RKCL5507

\title{
DETAILED BALANCE IN ION CHANNELS: APPLICATION OF FEINBERG'S THEOREM
}

\author{
Ilona Nagy ${ }^{\mathrm{a}}$, Benedek Kovács ${ }^{1}$ and János Tóth ${ }^{\mathrm{a}, \mathrm{b} *}$ \\ ${ }^{a}$ Department of Analysis, Budapest University of Technology and \\ Economics, Budapest, H-1111 Egry J. u. 1. Hungary \\ ${ }^{b}$ Laboratory for Chemical Kinetics of the Institute of Chemistry \\ Eötvös Loránd University, Budapest, Hungary
}

Received December 19, 2008, in revised form January 8, 2009, accepted January 9, 2009

\begin{abstract}
Detailed balance helps reduce the number of necessary measurements on ion channel models. Even imprecise formulations may lead to correct conditions because of the special structure of these models.

Keywords: Ion channel, detailed balance, microscopic reversibility, deficiency, circuits, spanning forest
\end{abstract}

\section{INTRODUCTION}

Ion channels that help establish and control the voltage gradient across the membrane of all living cells by allowing the flow of ions against their electrochemical gradient are present everywhere in living organisms and they play an especially important role in the nervous system [1, 2]. Models of ion channels are usually based on reaction kinetics: they are either deterministic [2] or stochastic [3] models. Models of reaction kinetics may or may not obey the principle of detailed balance [4] (also called microscopic reversibility). According to usual belief [5] (and vague allusions to thermodynamics), only models obeying this principle are realistic, however, such useful and ubiquitous formal models as the triangle reaction, the Lotka-Volterra model, the Brusselator, Explodator, Oregonator do not have this property.

The practical importance of detailed balance in deterministic kinetics comes from the fact that it implies a set of equalities between products of powers of

* Corresponding author. E-mail: jtoth@math.bme.hu 
reaction rate coefficients. From the point of view of experimentalists, these equalities allow a decrease in the number of necessary independent measurements: some of the rate coefficients can be calculated from these equalities considered as equations for them [2]. That is why Colquhoun et al. [2] proposed a method to construct the necessary and sufficient conditions of detailed balance in the case of ion channels in form of equalities.

However, they used a kind of notation to describe reaction steps which is not detailed enough (neglecting mass conservation) but can easily be transformed into the correct form behind their arguments. The major problem is that they considered (quite in accordance with the usual belief among chemists) the circuit conditions to be not only necessary but also sufficient as well.

We reformulated a series of models, including theirs in a form not contradicting to mass conservation and wrote down the correct equalities necessary and sufficient for detailed balancing: not only the circuit conditions but also the spanning forest conditions ${ }^{1}$. Good intuition of the mentioned authors is reflected in the results: namely, in all of the examples (altogether five) investigated by now, the results obtained by the two methods are the same. Here, we present one example and one virtual counterexample. Our general statement to be outlined in a forthcoming detailed paper is that ion channel models are of very special structure to allow such a reformulation that although the equalities in the correct formulation are of different origin, finally they lead to the same relations and the same number of necessary measurements as those proposed by Colquhoun et al. [2].

\section{THEORETICAL}

We introduce the definitions on the following example:

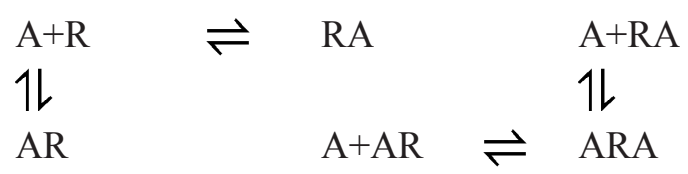

In the above reaction, there are $M=5$ chemical species: 1 . A, 2. R, 3. AR, 4 . RA, 5. ARA; $N=6$ complexes: 1. AR, 2. A+R, 3. RA, 4. A+RA, 5. ARA, 6. $\mathrm{A}+\mathrm{AR}$. The complexes can also be described by the complex vectors:

$$
\mathbf{x}_{1}=(0,0,1,0,0), \mathbf{x}_{2}=(1,1,0,0,0), \mathbf{x}_{3}=(0,0,0,1,0),
$$

\footnotetext{
${ }^{1}$ The terms circuit conditions in [4] and spanning tree method in [2] mean the same.
} 


$$
\mathbf{x}_{4}=(1,0,0,1,0), \mathbf{x}_{5}=(0,0,0,0,1), \mathbf{x}_{6}=(1,0,1,0,0)
$$

showing the quantity of the individual species forming the complexes. The effect of the reaction steps can be described by the reaction vectors, such as $\mathbf{v}_{12}=\mathbf{x}_{2}-\mathbf{x}_{1}=(1,1,-1,0,0)$, which give the net result of the step with reaction rate coefficient $k_{12}$. The stoichiometric space is the linear space generated by the reaction rate vectors, its dimension, $S$, is 3 in our case. The graph above, the Feinberg-Horn-Jackson graph of the reaction consists of two connected components, or linkage classes, therefore the number $L$ of linkage classes is 2 . The deficiency $\delta$, a nonnegative integer characterizing the reaction is defined to be $\delta:=N-L-S=6-2-3=1$. Our reaction is reversible, and the number of the reaction pairs is $P=4$. The spannig forest of the Feinberg-Horn-Jackson graph is any directed forest (union of disjoint trees) containing all the vertices of the graph, in our case e. g.

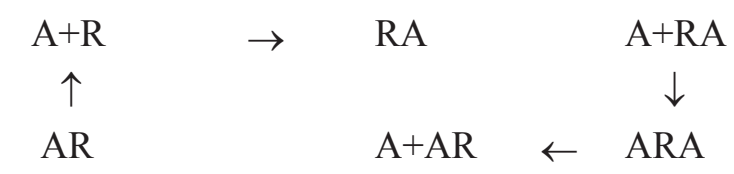

Let us consider another related graph (where the complexes are numbered as 1 . ARA, 2. AR, 3. R, 4. RA), and disregard the fact that this reaction does not seem to obey the law of mass conservation.

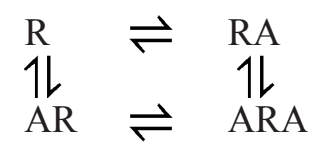

Here, the corresponding quantities are: $M=4, N=4, L=1, S=3, \delta=0$. This reaction is not only reversible but its graph also contains a circuit.

We only consider mass action type kinetics here. Suppose the kinetic differential equation of a reaction is $\dot{\mathbf{c}}(t)=\mathbf{f}(\mathbf{c}(t))$. The concentration vector $\mathbf{c}^{*}>\mathbf{0}$ is said to be a stationary point, if $\mathbf{0}=\mathbf{f}\left(\mathbf{c}^{*}\right)$. A reversible reaction is detailed balanced at the stationary point $\mathbf{c}^{*}$, if all the reaction steps have the same rate at the stationary point. It is detailed balanced, if it is detailed balanced at all the stationary points. Feinberg [4] provided two sets of conditions which together form a necessary and sufficient condition for detailed balancing. 
The circuit conditions require that the product of the reaction rate coefficients should be the same along all circuits, if taken in either directions. (Of all the circuits it is enough to take any of the $P-(N-L)$ independent circuits.)

The spanning forest conditions require the equalities of power products of the reaction rate coefficients obtained in the following way. Let $\alpha_{1}, \alpha_{2}, \ldots, \alpha_{\delta}$ be the independent solutions of the equation $\sum_{(i, j)} \alpha(i, j) \cdot \mathbf{v}_{i j}=\mathbf{0}$ (where the sum is taken for all reaction vectors $v_{i j}$ ). Then the following equalities should hold: $\prod k_{i j}^{\alpha_{i j}}=\prod k_{j i}^{\alpha_{i j}}$ where the products are taken for all reaction steps in the spanning forest.

Three trivial but important consequences of Feinberg's theorem are:

If $\delta=0$, the circuit conditions alone are necessary and sufficient.

For circuitless reactions, the spanning forest conditions alone are necessary and sufficient.

If $\delta=0$ and there is no circuit in the Feinberg-Horn-Jackson graph of the reaction, then the reaction is unconditionally (i. e. regardless of the values of the reaction rate coefficients) detailed balanced.

The reason why most people in the chemist community believe that the circuit conditions (also called Wegscheider conditions here and Kolmogorov conditions in the theory of stochastic processes) are necessary and sufficient is that most of the models in textbooks are of deficiency zero.

\section{RESULTS AND DISCUSSION}

Actually, model (2) is a simple ion channel model presented by Colquhoun [2], with $\mathrm{R}$ as a receptor and $\mathrm{A}$ as a ligand. Following his procedure the conditions of detailed balancing are:

$$
k_{12} k_{23} k_{34} k_{41}=k_{21} k_{14} k_{43} k_{32},
$$

where $k_{i j}$ denotes the rate coefficient of the reaction $i T j$.

Model (1) is an "improved" version in as much as it reflects mass conservation. Following the correct procedure given by Feinberg gives: there are no circles but the deficiency being zero we have a single equation. To write down this, we need the reaction vectors corresponding to the spanning forest:

$$
\begin{aligned}
& \mathbf{v}_{12}=(1,1,-1,0,0), \mathbf{v}_{23}=(-1,-1,0,1,0), \\
& \mathbf{v}_{45}=(-1,0,0,-1,1), \mathbf{v}_{56}=(1,0,1,0,-1),
\end{aligned}
$$


therefore we have to find one solution to the equation

$\alpha(1,1,-1,0,0)+\beta(-1,-1,0,1,0)+\gamma(-1,0,0,-1,1)+\delta(1,0,1,0,-1)=0$.

As this may be chosen to be $\alpha=\beta=\gamma=\delta=1$, the condition is literally the same as above.

What is the problem with Colquhoun's approach then? If we consider the simplest example by Feinberg [4] and apply the two different methods to check detailed balance, not even the number of equations will be the same in the two cases. The reason of this difference is not mathematical: the form of ion channel models cannot be arbitrary, and once we restrict ourselves to the class of models which can occur as ion channel models the two methods will lead to equivalent results. This will be proved in our forthcoming paper in detail, here we give a few hints.

Our method of proving the equivalence of the two approaches consists of a transformation leading from the original graph of the model of an ion channel to a mass conserving model. Our observations on all the examples are summarized in more specific statements:

There is no cycle in the transformed system.

The stoichiometric space is of the same dimension in the transformed system as in the original one: $S=S^{\prime}$.

If the number of independent cycles in the original system is $k$, then $\left(N^{\prime}-N\right)-\left(L^{\prime}-L\right)=\delta^{\prime}=k$.

Finally, the circuit conditions in the original system are equivalent to the spanning forest condition in the transformed system.

An immediate consequence of the statements above is that of the $2 P$ reaction rate coefficients only $2 P-\delta-k$ are to be measured.

Discussion with P. Érdi of an earlier version of the present paper, and collection of examples by A. Szabó is acknowledged.

\section{REFERENCES}

1. M. Arbib, P. Érdi, J. Szentágothai: Neural Organization: Structure, Function and Dynamics, The MIT Press, p. 124. Cambridge-London 1997.

2. D. Colquhoun, K.A. Dowsland, M. Beato, A.J.R. Plested: J. Biophys., 86, 3510 (2004).

3. P. Érdi, L. Ropolyi: Biol. Cyb., 32, 41 (1979).

4. M. Feinberg: Chem. Eng. Sci., 44, 1819 (1989).

5. http://en.wikipedia.org/wiki/Microscopic_reversibility 